\title{
Quality Factor Maximization Through Dynamic Balancing of Tuning Fork Resonator
}

\author{
Sergei A. Zotov, Member, IEEE, Brenton R. Simon, Member, IEEE, Igor P. Prikhodko, Member, IEEE, \\ Alexander A. Trusov, Member, IEEE, and Andrei M. Shkel, Fellow, IEEE
}

\begin{abstract}
This paper presents a method of dynamically balancing tuning fork microresonators, enabling maximization of quality factor ( $Q$-factor) in structures with imperfections. Nonsymmetric tuning of stiffness in a coupled 2-DOF resonator is completed through the use of the negative electrostatic spring effect. This variable stiffness is shown to be able to adjust the reaction forces of the structure at the anchors, effectively balancing any spring imperfections caused by fabrication imperfections. Balancing the structure through stiffness matching minimizes the loss of energy through the substrate and maximizes $Q$-factor of the device's antiphase mode. The approach is experimentally demonstrated using a vacuum packaged microelectromechanical tuning fork resonator with operational frequency of $2.2 \mathrm{kHz}$ and antiphase $Q$-factor of 0.6 million. By electrostatically tuning the reaction force at the anchors caused by fabrication imperfections, anchor loss can be suppressed, increasing the $Q$-factor to above 0.8 million. The experimentally validated analytical model of substrate dissipation is confirmed to be applicable to $Q$-factor tuning in antiphase driven resonators and gyroscopes.
\end{abstract}

Index Terms-Q-factor, tuning fork resonator, MEMS.

\section{INTRODUCTION}

$\mathbf{M}$ AXIMIZATION of the mechanical quality factor is critical for improving the performance of micromachined vibratory transducers [1]. Resonant accelerometers [2], vibratory gyroscopes [3], [4], RF filters [5], and resonant chemical sensors [6] all benefit from high Q-factor architectures [7]. Energy dissipation in vibratory MicroElectroMechanical Systems (MEMS) is governed by several mechanisms, including viscous damping, dissipation through the substrate (anchor loss), Thermo-Elastic Dissipation (TED), and resonator surface effects [8]. When air damping is minimized through vacuum packaging, the device Q-factor is typically limited by the substrate dissipation and thermoelastic damping for the studied frequency range [9], [10].

Tuning fork structures are widely used as resonators due to a number of advantages, such as minimized substrate

Manuscript received January 8, 2014; revised March 18, 2014; accepted March 19, 2014. Date of publication April 2, 2014; date of current version July 1, 2014. This work was supported by the Office of Naval Research/Naval Surface Warfare Center Dahlgren Division under Grant N00014-09-1-0424 and Grant N00014-11-1-0483. The associate editor coordinating the review of this paper and approving it for publication was Dr. Stefan J. Rupitsch.

The authors are with the MicroSystems Laboratory, Department of Mechanical and Aerospace Engineering, University of California at Irvine, Irvine, CA 92697 USA (e-mail: szotov@uci.edu; brsimon@uci.edu; iprikhod@uci.edu; atrusov@uci.edu; ashkel@uci.edu).

Color versions of one or more of the figures in this paper are available online at http://ieeexplore.ieee.org.

Digital Object Identifier 10.1109/JSEN.2014.2314614



Fig. 1. Concept of anti-phase Q-factor tuning: matching tine stiffness eliminates anchor loss and maximizes Q-factor up to the thermoelastic limit of the structure.

energy dissipation and common-mode rejection of acceleration [1], [11]-[14]. An ideally balanced tuning fork resonator is comprised of two coupled tines, each driven in opposite directions, or anti-phase resonance. This mode of resonance minimizes the net reaction force applied to the substrate, providing both rejection of common-mode external accelerations [15], as well as reduction of energy dissipation from the vibrating structure [16]. However, imperfections are commonplace in MEMS fabrication technologies, which prevent ideal antiphase motion. These imperfections create variances in the nominal mass and stiffness values, causing structural asymmetries in the final micromachined device. Despite driving the resonator in anti-phase motion, this structural asymmetry leaks energy through the substrate due to a vector force. This causes the dissipation of energy through the substrate for the anti-phase mode [16], as well as an undesired susceptibility to common-mode acceleration.

Recently, we have reported a method for the maximization of Q-factor for anti-phase driven tuning fork MEMS using the negative electrostatic spring effect [17]. Work [18] has also presented empirical evidence on the effect of polarization voltage on Q-factor in multi-beam tuning forks.

This paper demonstrates that energy loss can be minimized post-fabrication by matching the suspension stiffness of the two tines via a voltage-controlled, negative electrostatic spring, Figure 1. We present an analytical method for tuning the momentum imbalance in tuning fork micro-resonators and provide an experimental analysis of the effect on anti-phase tuning forks [17].

This paper comprises six sections. A description of the tuning fork resonator architecture with associated mathematical model is presented in Section II. This model is then 




Fig. 2. Optical photograph of a dual-mass anti-phase resonator used for the experimental study of substrate dissipation and Q-factor tuning.

evaluated by Finite-Element Analysis (FEA) of thermo-elastic damping and anchor loss, with results presented in Section III. Section IV presents a method of Q-factor regulation based on stiffness matching through the negative electrostatic spring effect, while Section V details recommendations to avoid anchor loss during resonator design. Sections VI concludes this paper with a discussion and summary of the results.

\section{Theoretical Model for Substrate Dissipation}

This section presents a mathematical model for substrate dissipation in non-ideal tuning forks. The model quantitatively explains the effect of structural imbalance on Q-factor.

\section{A. Tuning Fork Resonator Architecture}

A low-dissipation, dual-mass, tuning fork resonator [15], fabricated using a single Silicon-on-Insulator (SOI) wafer as the substrate, insulator, and device layer (100 microns thick), is used for the Q-factor study, Figure 2. This resonator is formed by two linearly-coupled tines actuated in anti-phase, Figure 3. Each tine is anchored to the substrate using shuttles to decouple the two-dimensional motion into single axes, where all electrostatic actuation, detection, and tuning takes place on these decoupled shuttles. A total of 16 springs anchor each tine: one spring to attach the tine to the shuttle, one spring to attach the shuttle to the anchor, and repeated a total of 8 times for each corner of the device and each axis of motion. Each spring is identical, comprised of a hairpin design, each beam having a length of 500 microns and width of 10 microns. The shuttles between each tine are then coupled with a double U-shaped spring, each beam having a length of 1400 microns and width of 15 microns. The proposed architecture prioritizes the Q-factor of the resonator through mechanical design, where the anti-phase oscillations is nominally balanced in both vector force and angular momentum, in order to minimize the dissipation of energy through the substrate. This tuning fork resonator is designed to be driven in the anti-phase mode of resonance.

In addition to the mechanical structure, each shuttle has differential comb electrodes for actuation or detection of the motion of each tine individually. There are also two parallel

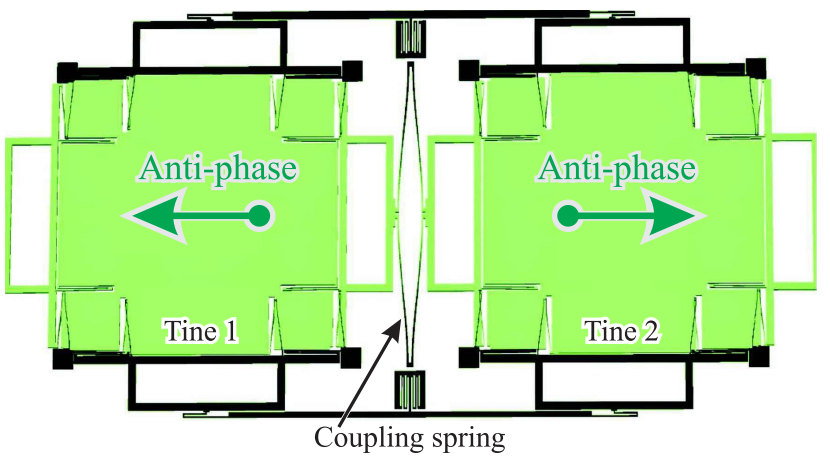

(a)

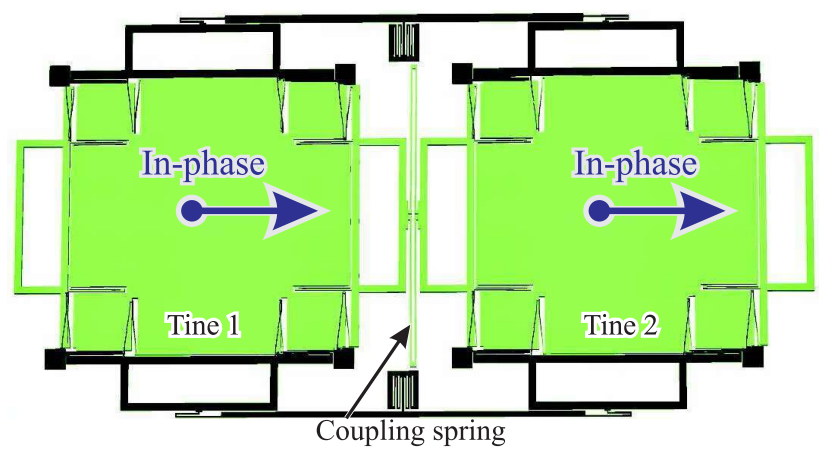

(b)

Fig. 3. FEM modeling of the dual mass tuning fork gyroscope sense-mode, illustrating the main anti-phase and undesired in-phase motion (parallel plate electrodes for tuning are not shown). (a) Linearly coupled anti-phase sensemode with dynamic balance of reaction force and moment for Q maximization. (b) Linearly coupled in-phase low-Q mode.

plate tuning electrodes on either side of the combs. Each tuning electrode consists of 8 beams, 500 microns long, with a working gap of 7 microns and anti-gap of 21 microns.

\section{B. Equations of Motion}

In this section the equations of motion are written under the following assumptions: 1) Each tine is a rigid body with equal mass, 2) The mass of the springs are negligible in comparison with the mass of tines, and 3) stiffness mismatches of the springs due to fabrication imperfections are small in comparison with the nominal stiffness value.

Figure 4 shows a conceptual schematic used to analytically describe effects of the stiffness tuning process on the dissipation of energy through the substrate. The masses are free to resonate along an axis collinear to their geometric orientation, which we define as the $x$-axis. This axis is displayed horizontally in Figure 4. Positions of the left and right tines of the tuning fork along the $x$-axis are described by coordinates $x_{1}$ and $x_{2}$, respectively. The equations of motion for this system can be written as

$$
\begin{aligned}
& m_{1} \ddot{x}_{1}+c \dot{x_{1}}+\left(k_{1}+k_{12}\right) x_{1}-k_{12} x_{2}=F(t), \\
& m_{2} \ddot{x}_{2}+c \dot{x_{2}}+\left(k_{12}+k_{2}\right) x_{2}-k_{12} x_{1}=-F(t),
\end{aligned}
$$

where $k_{1}, k_{2}, k_{12}$ are the stiffnesses of the springs; $c$ is the absolute damping coefficient, Figure 4; and $F(t)$ is the antiphase excitation force. 




Fig. 4. Schematic of the Q-factor regulation in a tuning fork resonator based on electrostatic shifting of stiffnesses. Coordinates $x_{1}$ and $x_{2}$ represent the displacement of the left and right tines with respect to their equilibrium position.

Assuming the tines are initially matched in mass $\left(m_{1}=\right.$ $\left.m_{2}=m\right)$, a new coordinate system is introduced $\left(y_{i n}, y_{a n}\right)$, where $y_{\text {in }}=\left(x_{1}+x_{2}\right) / 2$ and represents the amount of the undesired in-phase motion, while the amount of the primary anti-phase motion is given by $y_{a n}=\left(x_{1}-x_{2}\right) / 2$. Equations of motion of the tuning fork can be written as

$$
\begin{aligned}
\ddot{y}_{a n}+\frac{\omega_{a n}}{Q_{a n}} \dot{y}_{a n}+\omega_{a n}^{2} y_{a n} & =\frac{\Delta k y_{i n}}{2 m}+\frac{F(t)}{m}, \\
\ddot{y}_{i n}+\frac{\omega_{i n}}{Q_{i n}} \dot{y}_{i n}+\omega_{i n}^{2} y_{i n} & =\frac{\Delta k y_{a n}}{2 m} ;
\end{aligned}
$$

where $\omega_{i n}=\sqrt{\left(k_{1}+k_{2}\right) / 2 m}, \omega_{a n}=\sqrt{\left(k_{1}+k_{2}+4 k_{12}\right) / 2 m}$ and $Q_{i n}=m \omega_{i n} / c, Q_{a n}=m \omega_{a n} / c$ are the decoupled natural frequencies and Q-factors of the ideal anti-phase and in-phase motion, respectively, and $\Delta k=k_{2}-k_{1}$ is the stiffness mismatch, Figure 4.

Fabrication imperfections cause asymmetry of the MEMS resonator suspension, represented by stiffness mismatches, $\Delta k$. Spring stiffness is proportional to its width cubed, which results in a high sensitivity to variations, defined by lithography and the etching process. This work studies analytically and experimentally the anti-phase motion of nominally matched tuning fork tines with imbalances $\left(\Delta k \ll k_{1}\right.$, and $\left.\Delta k \ll k_{2}\right)$.

\section{Equations of Motion Analysis}

The tuning fork resonator is designed to be driven in the anti-phase resonance. We consider the case when the excitation force $F(t)$, equation (2), is harmonic and frequency is equal to $\omega_{a n}$ with a constant amplitude. Also, we assume that the system has been observed long enough to reach steadystate. In this case, the anti-phase component, $y_{a n}$, is given by a sinusoid of amplitude $A_{a n}$ and frequency $\omega_{a n}$ :

$$
y_{a n}=A_{a n} \sin \left(\omega_{a n} t\right) .
$$

Substituting equation (3) in (2), the steady-state solution for the in-phase component of motion $y_{i n}$ becomes [19]

$$
y_{i n}=A_{a n} \frac{\Delta k \sin \left(\omega_{a n} t+\phi\right)}{2 m \sqrt{\left(\omega_{i n}^{2}-\omega_{a n}^{2}\right)^{2}+\omega_{i n}^{2} \omega_{a n}^{2} / Q_{i n}^{2}}} .
$$

Analytical solutions (3) and (4) with equations (2) will be used to analyze the effect of anchor loss on the anti-phase Q-factor.

\section{Effect of Anchor Loss on Anti-Phase Q-Factor}

In order to derive the analytical solution of energy dissipation through the substrate due to imbalances in the tuning fork resonator, the following physical laws are used:

1) Instantaneous power of the system is a product of force and velocity;

2) The dissipation of energy per cycle of the anti-phase harmonic motion with period $\mathrm{T}=\frac{2 \pi}{\omega_{a n}}$ is given by an integral of the system power defined over a full time period (from 0 to $2 \pi / \omega_{a n}$ ) [20].

Also note that the harmonic functions $\ddot{y}$ and $\dot{y}$, as well as functions $y$ and $\dot{y}$, are orthogonal. This means the integral of the product of these functions, defined over a full period, is equal to 0 . Accordingly, using solution (3) and equation (2), the dissipation of energy per cycle of the antiphase motion, $\Delta E_{a n}$, is given by an integral of the dissipative term $m \omega_{a n} \dot{y}_{a n} / Q_{a n}$ multiplied by the anti-phase velocity $\dot{y}_{a n}$.

$$
\Delta E_{a n}=\int_{0}^{2 \pi / \omega_{a n}} m \frac{\omega_{a n}}{Q_{a n}} \dot{y}_{a n}^{2} d t=m A_{a n}^{2} \pi \frac{\omega_{a n}^{2}}{Q_{a n}},
$$

where it is assumed that $\Delta k \ll k_{1}, \Delta k \ll k_{2}$, and $\left\|y_{i n}\right\| \ll\left\|y_{\text {an }}\right\|$. Similarly, the energy dissipated per cycle for the in-phase mode is calculated as

$$
\begin{aligned}
\Delta E_{i n} & =\int_{0}^{2 \pi / \omega_{a n}} m \frac{\omega_{i n}}{Q_{i n}} \dot{y}_{i n}^{2} d t \\
& =\pi \frac{A_{a n}^{2}}{Q_{i n}} \frac{\Delta k^{2} \omega_{i n} \omega_{a n}}{4 m\left(\left(\omega_{i n}^{2}-\omega_{a n}^{2}\right)^{2}+\omega_{i n}^{2} \omega_{a n}^{2} / Q_{i n}^{2}\right)} .
\end{aligned}
$$

At the same time, the total mechanical energy in the system can be approximated by the energy stored within the anti-phase mode of vibration:

$$
E_{\text {Stored }}=\frac{1}{2} m\left(A_{a n} \omega_{a n}\right)^{2} .
$$

Finally, the quality factor is defined as the ratio between stored and dissipated energy over one cycle of vibration, multiplied by $2 \pi$ :

$$
Q=2 \pi \frac{\text { Energy stored }}{\text { Energy dissipated per cycle }} .
$$

where the anti-phase quality factor corresponding to the dissipation of energy through the substrate due to structural imbalances, $Q_{\Delta k}$, can now be found by substituting the energy expressions (6) and (7) into the given Q-factor definition (8):

$$
Q_{\Delta k}=\frac{A_{\Delta k}}{\Delta k^{2}}
$$

where

$$
A_{\Delta k}=Q_{i n} 4 m^{2} \frac{\omega_{a n}\left(\left(\omega_{i n}^{2}-\omega_{a n}^{2}\right)^{2}+\omega_{i n}^{2} \omega_{a n}^{2} / Q_{i n}^{2}\right)}{\omega_{i n}} .
$$


The energy dissipation through the substrate due to fabrication imperfections is inversely proportional to the stiffness mismatch squared (9) with coefficient (10), and depends on the in-phase and anti-phase natural frequencies.

Note here that as previously calculated in Equation (9), Q-factor, $Q_{\Delta k}$, is defined as the energy loss through the substrate due to fabrication imperfections. As $\Delta k$ increases, the coupling between the anti-phase and in-phase modes also increases, resulting in additional energy loss from the mode with higher Q-factor: the anti-phase resonance. The total Q-factor of the anti-phase mode can be calculated by combining the various loss mechanisms [8]:

$$
Q_{\text {Total }}=\left(\frac{1}{Q_{T E D}^{\text {anti }}}+\frac{1}{Q_{\text {Anchor }}^{\text {anti }}}+\frac{1}{Q_{\Delta k}}\right)^{-1},
$$

where: $Q_{T E D}^{a n t i}$ and $Q_{\text {Anchor }}^{a n t i}$ are Q-factors defining the energy loss through the anchors and thermoelastic damping (TED). Numerical calculation of the anchor loss and TED for the anti-phase mode are provided in the following section.

\section{Finite Element AnAlysis And ANALYTiCAl Modeling}

While many loss mechanisms have been reported, viscous damping traditionally dominates on the micro-scale. To alleviate this effect, the tested device has been vacuum-sealed with getter material. As a result, additional loss mechanisms become dominate, and for devices in similar scale and frequency as reported here, this has been observed to be anchor losses and thermoelastic damping [15].

In this section, quality factor for a vacuum-packaged, ideally balanced tuning fork structure is calculated for both its antiphase, $Q_{a n}$, and in-phase, $Q_{i n}$, resonances by using finite element modeling to predict the influence of the new dominate loss mechanisms. This is completed through modal analysis using COMSOL Multiphysics software.

In both cases, fabrication was completed using a standard (100) silicon wafer orientation, with devices aligned to the wafer flat. The entire device was modeled with a density of $2330 \mathrm{~kg} / \mathrm{m}^{3}$, a constant Young's Modulus of $170 \mathrm{GPa}$ [21], thermal expansion coefficient of $2.6 \mathrm{ppm} / \mathrm{K}$, and a static mesh consisting of triangular/tetrahedral elements, Figure 5(a), 7(a).

\section{A. Thermoelastic Limit of Q-Factor}

Thermo-Elastic Damping (TED) [22], [23] was determined based on a 2-D model of the device and resulted in Q-factors of $Q_{T E D}^{\text {anti }}=1.42 \times 10^{6}$ and $Q_{T E D}^{i n}=1.62 \times 10^{6}$ for the anti-phase and in-phase modes, respectively, Figure 5(b). This Q-factor converged to the reported values as the mesh elements number were increased from $21 \times 10^{3}$ to $63 \times 10^{3}$, confirming convergence of the model with a 5\% tolerance, Figure 5(b). This type of damping only takes into account the energy loss due to thermal gradient induced by stress within flexures of the structure, and is specifically limited by the springs anchoring each tine to the substrate. Images of the stress distribution and temperature gradient induced by this motion is shown in Figure 6. Due to the additional stiffness of the coupling

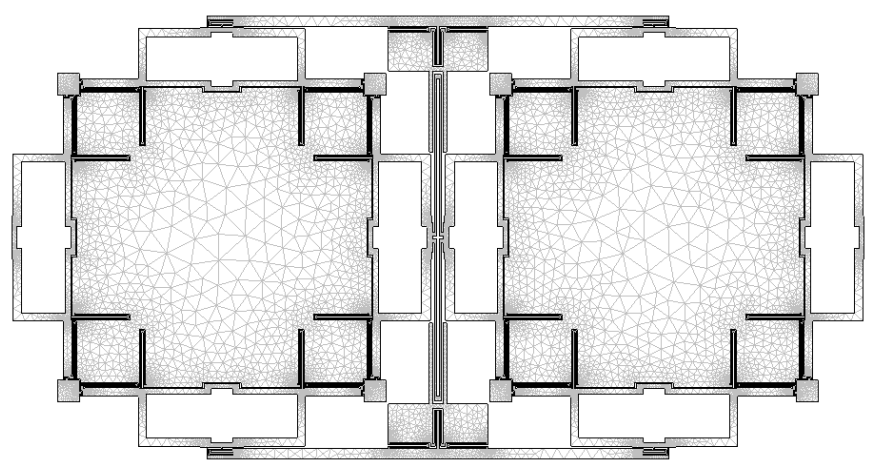

(a)

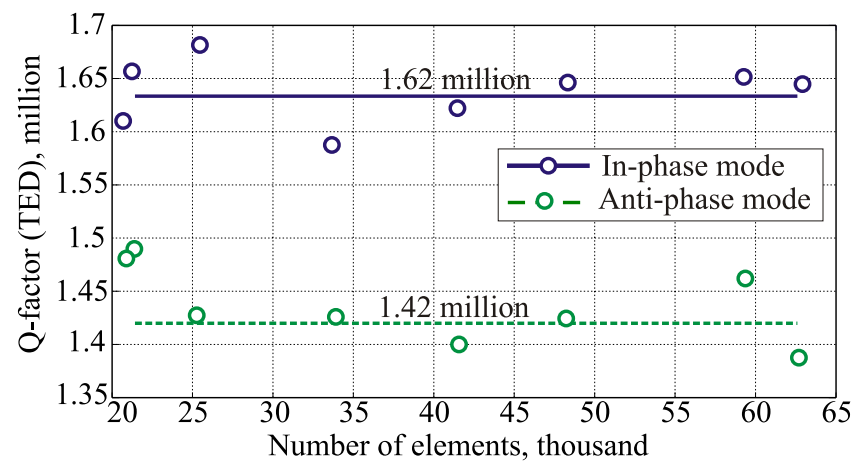

(b)

Fig. 5. FEM for thermoelastic damping yields a Q-factor of the anti-phase mode of $1.42 \times 10^{6}$ and Q-factor of the in-phase mode of $1.62 \times 10^{6}$. (a) 2-D FEM mesh for thermoelastic model. (b) Dependence of Q-factors for in-phase and anti-phase modes on the number of mesh elements.

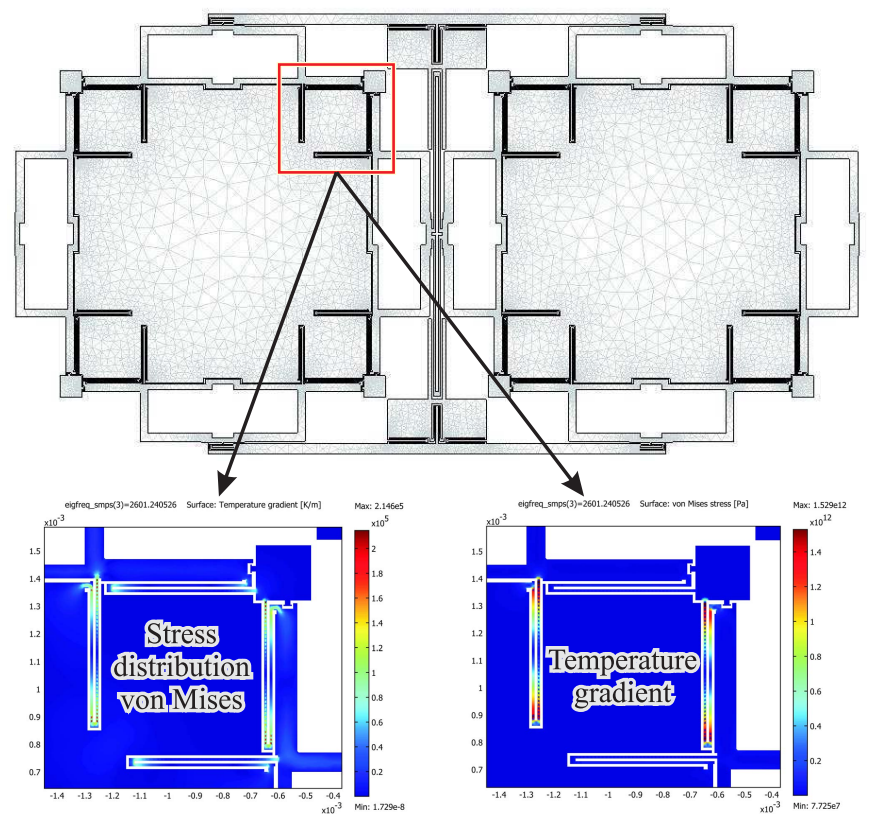

Fig. 6. Stress distribution (left) and the generated temperature gradiant (right) of the gyroscope's springs during the TED analysis.

spring during anti-phase resonance, Figure 3(a), stress within this vibratory mode is larger than the in-phase resonance, Figure 3(b). 




(a)

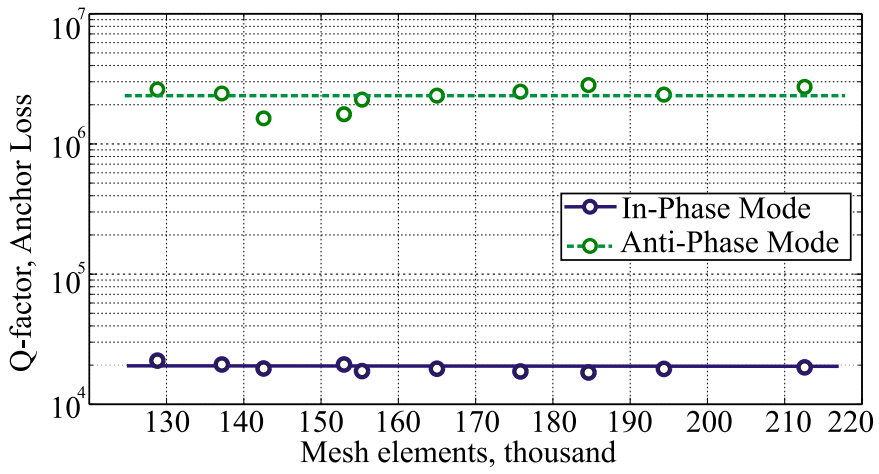

(b)

Fig. 7. FEM for energy dissipation through substrate (anchor loss) result in Q-factor of the anti-phase mode of $2.4 \times 10^{6}$ and Q-factor of in-phase mode of $17 \times 10^{3}$. (a) 3-D mesh of anchor loss model, highlighting PML layer for modeling. (b) Dependence of Q-factors for in-phase and anti-phase modes on the number of elements.

\section{B. Anchor Loss Limit of Q-Factor}

Anchor losses were computed with a 3-D FEA model containing the device, substrate, and a Perfectly Matched Layer (PML) [24]. The PML represents an infinite boundary, behaving as an acoustic absorption layer. Acoustic waves that enter the PML attenuate before they can be reflected back into the model, Figure 7(a). The footprint of the substrate is $8.2 \times 8.2 \mathrm{~mm}$. In this study, a PML beneath the substrate is chosen to absorb one wavelength of stress, transmitted at the resonant frequency of the vibratory modes. The Q-factor of the in-phase mode is calculated to be $Q_{\text {Anchor }}^{\text {in }}=1.8 \times 10^{4}$, while the anti-phase mode is $Q_{\text {Anchor }}^{\text {anti }}=2.4 \times 10^{6}$. Mesh elements, varied from $1.29 \times 10^{5}$ to $2.12 \times 10^{5}$, confirm the convergence of the model with a tolerance of 5\%, Figure 7 .

\section{Total Quality Factor}

With both loss mechanisms taken into account, numerical modeling demonstrates that the in-phase mode is anchor loss limited with an effective Q-factor of

$$
Q_{\text {in }}=\left(\frac{1}{Q_{T E D}^{i n}}+\frac{1}{Q_{\text {Anchor }}^{i n}}\right)^{-1}=18,000 .
$$

Similarly for the ideally balanced anti-phase mode:

$$
Q_{a n}=\left(\frac{1}{Q_{T E D}^{\text {anti }}}+\frac{1}{Q_{\text {Anchor }}^{\text {anti }}}\right)^{-1}=890,000 .
$$



Fig. 8. Schematic of the Q-factor tuning characterization experiment using a vacuum packaged dual mass gyroscope and a laser vibrometer.

For the non-ideal anti-phase motion, the total Q-factor can be represented by:

$$
Q_{\text {Total }}=\left(\frac{1}{Q_{a n}}+\frac{1}{Q_{\Delta k}}\right)^{-1},
$$

where $Q_{\Delta k}$ is the $\mathrm{Q}$-factor corresponding to the anchor loss due to structural imbalances [see equation (9)].

Expressions (9), (10), and (13) constitute a mathematical model of the dominant effects for the energy dissipation in non-ideal tuning fork resonators. In the next section, this mathematical model is compared to experimental data in order to extract device parameters, such as stiffness mismatch, $\Delta k$, which is difficult to measure directly or estimate analytically.

\section{ExPERIMENTAL CHARACTERIZATION}

This section describes the experimental investigation of the stiffness tuning effect on the substrate energy dissipation using the tested tuning fork resonator.

\section{A. Experimental Testbed}

The tuning fork resonator was excited into the anti-phase vibrations using a combination of constant DC polarization voltage and AC voltage generated by a PLL. A carrier of $1 \mathrm{Vrms}$ at $52 \mathrm{kHz}$ was applied to the proof mass, resulting in the amplitude modulation of the signal corresponding to the velocity of proof mass. The motional signal from the detection capacitors was amplified and demodulated twice: First at the carrier frequency, and a second time at the antiphase resonance frequency, in order to extract the amplitude of the anti-phase motion. To provide direct measurement of the substrate vibration, an optical method using a Polytec OVF-5000 single-point Laser Doppler Vibrometer was chosen. Specifically, the laser was focused at the edge of the die through an optical window in the vacuum package lid and used to directly measure the vibration of the substrate, Figure 8 . 


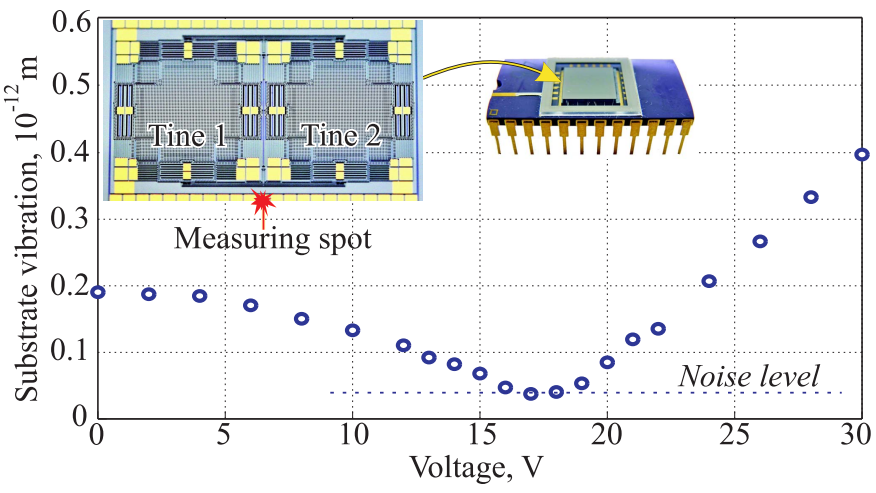

(a)

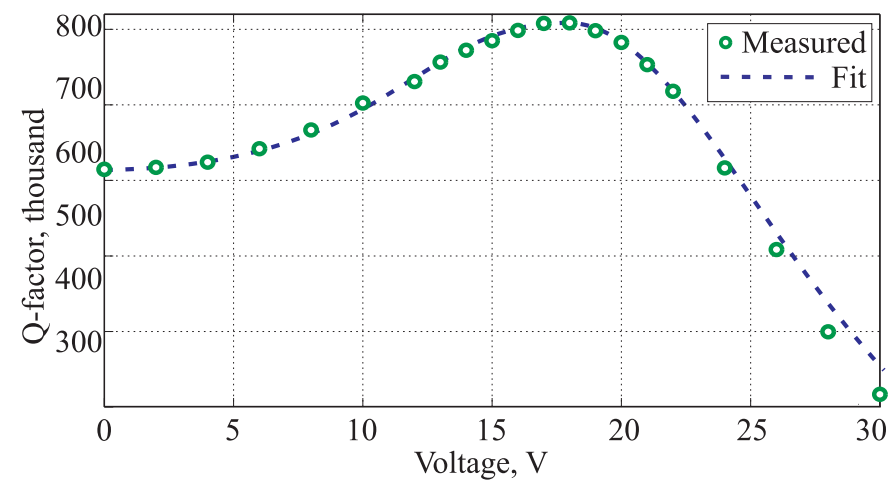

(b)

Fig. 9. Characterization of the stiffness matching effect on substrate vibration and gyroscope Q-factor (tine matching is achieved at $18 \mathrm{~V}$ ). (a) Measured substrate vibration for different tuning voltages. Stiffness matching minimizes transfer of energy to the substrate (anchor loss). (b) Measured Q-factor of the anti-phase mode. Tine stiffness matching maximizes Q-factor to 810,000 by minimizing the substrate loss.

The following procedure was used to determine the vibrational amplitude of the substrate, along with the Q-factor of the device for various values of a voltage-controlled negative electrostatic spring:

1) Apply a DC voltage to the stiffness-tuning parallel plate electrodes on one of the tines;

2) Electrostatically excite the anti-phase mode of the gyroscope into resonance with an amplitude of three microns;

3) Measure vibrational amplitude of the substrate die using the laser vibrometer;

4) Turn off the excitation voltage, record ring-down time history of free vibrations, and extract the Q-factor from the data;

5) Change the value of the stiffness tuning DC voltage and repeat steps (i)-(iv).

\section{B. Substrate Energy Dissipation and Q-Factor Tuning}

Measurements of the substrate vibration amplitude and device Q-factors for different values of tuning voltage are shown in Figure 9. Initially, the resonator has an anti-phase Q-factor of 0.6 million for a tuning voltage of 0 Volt, Figure 9(b); however, considerable substrate vibrations were detected by the vibrometer, showing an initial amplitude of $0.19 \mathrm{pm}$, Figure 9(a). Increasing the tuning voltage on the



Fig. 10. Measured anti-phase Q-factor versus substrate vibration, illustrating the effect of structural misbalance on substrate dissipation. (Using data from Figure 9.)

stiffer tine reduced the substrate vibrations, Figure 9(b), while simultaneously increasing Q-factor, Figure 9(a).

At approximately $18 \mathrm{~V}$ the minimal substrate vibration was revealed, which was on level with the hlnoise of the experimental setup. This same tuning voltage also corresponded to the observed Q-factor maximum of 0.81 million. Increasing the tuning voltage beyond $18 \mathrm{~V}$ resulted in a Q-factor reduction, as well as increased substrate vibration, thus increasing energy dissipation through the substrate, Figure 9.

The data from Figure 9 plotted in Figure 10 along axes of Q-factor versus substrate vibration, illustrates that minimization of anchor loss requires balancing of the tuning fork structure, Figure 10. The negative electrostatic spring effect allows for post-fabrication, voltage-controlled balancing of tuning fork resonators. The experiment achieved a quality factor of 0.81 million, which agrees with the limit of 0.89 million that was found using FEM, equation (13), thus agreeing with each other to within $10 \%$. The result confirms that the theoretical bulk-parameter model was able to capture the dominant source of energy loss accurately.

\section{Comparative Analysis of Modeled and Experimental Data}

The derived analytical model for energy dissipation in non-ideal tuning forks allows quantitative analysis of the experimental data, shown in Figure 9 and Figure 10. In these experiments, the effective stiffness mismatch $\Delta k_{\text {eff }}$ becomes a function of the negative electrostatic spring tuning voltage $(\mathrm{V})$ :

$$
\Delta k_{e f f}=\Delta k-\gamma V^{2},
$$

where $\gamma=\epsilon \frac{A}{g^{2}}$ is the coefficient defined by geometric parameters of the tuning electrodes: $g$ is the gap, and $A$ is the area of electrode overlap. The $\gamma$ can be experimentally determined by measuring the stiffness shift vs applied voltage, using either equation (2), that is $\omega_{i n}=\sqrt{\left(k_{1}+k_{2}-\gamma V^{2}\right) / 2 m}$, or $\omega_{a n}=\sqrt{\left(k_{1}+k_{2}+4 k_{12}-\gamma V^{2}\right) / 2 m}$.

Substituting (15) in (9) defines the solution for the effective Q-factor due to stiffness mismatch:

$$
Q_{\Delta k}^{e f f}=\frac{A_{\Delta k}}{\left(\Delta k-\gamma V^{2}\right)^{2}} .
$$

A parameter of the tuning fork resonator that is difficult to predict is the stiffness mismatch, $\Delta k$, which is due to 
TABLE I

TUNING FORK RESONATOR PARAMETERS

\begin{tabular}{|c|l|l|}
\hline Parameter & Value & Method \\
\hline \hline$\omega_{a n}$ & $2400 \mathrm{~Hz}$ & Measured \\
\hline$\omega_{i n}$ & $2340 \mathrm{~Hz}$ & Measured \\
\hline$Q_{i n}$ & 18,000 & Modeling (12) \\
\hline$Q_{a n}$ & 890,000 & Modeling (13) \\
\hline$Q_{a n}$ & 810,000 & Measured \\
\hline$\Delta k$ & $1.5 \%$ of $k_{1}$ & Data fit with model \\
\hline
\end{tabular}

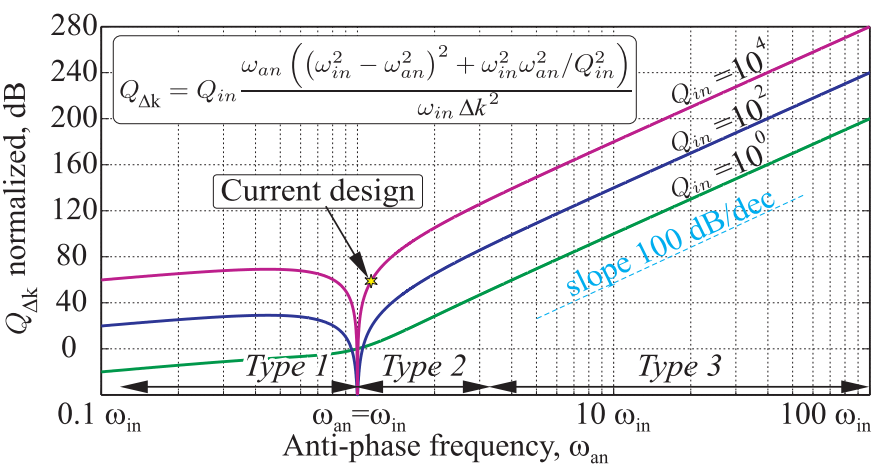

Fig. 11. Effects of the ratio between in-phase and anti-phase natural frequency on the dissipation of energy through the substrate due to stiffness mismatch.

fabrication imperfections. In order to estimate this parameter the experimental data was fitted to the analytical model (16), (13), (9). The parameter fitting procedure resulted in a good agreement, validating the developed bulk-parameter mathematical model, Figure 9(a), and Figure 10. Fitting of the data also allowed quantitative identification of the system imbalance. For the device under test, the relative stiffness mismatch $\Delta k$ of less than $1.5 \%$ was identified, the other parameters and the method of their detection is shown in Table I. The parameter $Q_{a n}$ was calculated using the analytical model, equation (13), and agrees with the empirically derived value with $10 \%$ accuracy.

\section{Anti-Phase Q-Factor Design Optimization}

Maximizing the Q-factor of the anti-phase mode, $Q_{\Delta k}$, implies maximizing equation (14). The analysis of the formula for $Q_{\Delta k}$, equations (9) and (10), reveals that energy dissipation is inversely proportional to the in-phase mode Q-factor, $Q_{i n}$, and proportional to the stiffness mismatches, $\Delta k$, squared, and multiplied by the coefficient $A_{\Delta k}$. This coefficient strongly depends on the in-phase and anti-phase frequencies equation (10). Therefore, for a fixed stiffness mismatch and constant parameter $\Delta k$, the value of energy dissipation through the substrate, $Q_{\Delta k}^{-1}$, strongly depends on the ratio between the in-phase and anti-phase natural frequencies, as well as on $Q_{i n}$. In order to show the effects of the natural frequency relationship on the dissipation of energy through the substrate, this coefficient is plotted in Figure 11 for three different values of $Q_{i n}$. Figure 11 shows that tuning fork resonators with a large separation between the resonant frequencies of the in-phase and anti-phase modes



(a)

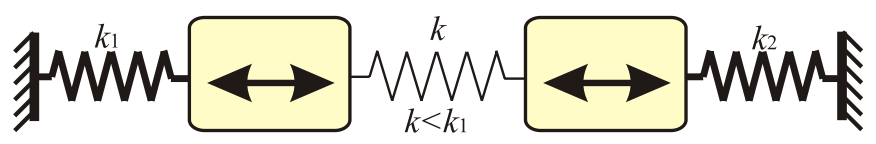

(b)

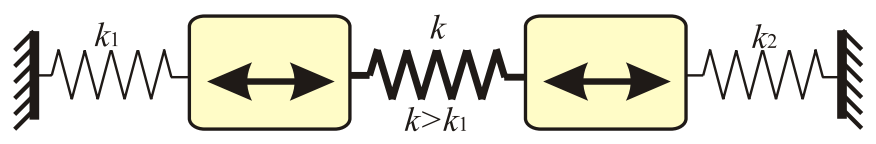

(c)

Fig. 12. The different types of coupling provides different dependence on the energy dissipation through the substrate due to stiffness mismatch. (a) Type 1: anti-phase frequency is less than in-phase. Anti-phase Q-factor is very sensitive due to fabrication imperfection [25]. (b) Type 2: anti-phase frequency is slightly larger than in-phase. Anti-phase Q-factor is sensitive due to fabrication imperfection. (c) Type 3: anti-phase frequency is significant larger than in-phase. Anti-phase Q-factor is almost not sensitive to fabrication imperfection. Trade off is low $Q_{T E D}$.

have a reduced dependence on the energy dissipation arising from stiffness mismatch. Increasing the $\omega_{i n} / \omega_{\text {an }}$ ratio is an effective design approach to reducing the energy dissipation in presence of fabrication imperfections with a trade off of decreasing $Q_{T E D}^{a n t i}$.

The ratio of the in-phase to anti-phase frequency of the device is solely determined by the coupling structure between the two masses. A descriptive example showing how this frequency ratio can be manipulated is shown in Figure 12, through the use of three different resonator designs. The first resonator, Figure 12(a), has a coupling spring that effectively creates a high stiffness for in-phase motion and low stiffness for anti-phase motion [25]. This design allows inverting the in-phase and anti-phase modes, such that $\left(\omega_{i n}>\omega_{a n}\right)$. The second resonator, Figure 12(b), has a weak coupling spring making the two modes of resonance close in natural frequency $\left(\omega_{i n}<\omega_{a n}\right)$. Both of these designs suffer from a high sensitivity of Q-factor on fabrication imperfection. The third resonator, Figure 12(c), contains a strong coupling spring, which makes the ratio between in-phase and anti-phase natural frequency large $\left(\omega_{i n} \ll \omega_{a n}\right)$. This reduces the sensitivity of Q-factor to fabrication imperfections, reducing the energy loss for even larger stiffness mismatch between the tine springs. However, since the anti-phase frequency of the third design is very high, it leads to the lowering of thermoelastic damping, $Q_{T E D}$. Increasing the stiffness of the coupling spring will have no effect on TED of the in-phase mode, as the spring does not flex for this mode of resonance. The coupling spring will, however, affect the anti-phase mode by increasing the mechanical resonance frequency.

In summary, there are three design approaches to managing the anchor loss energy dissipation:

1) Increase the $\mathrm{Q}$-factor of in-phase mode $\left(Q_{i n}\right)$, using rigid die attachment [16]. 
2) Reduce the stiffness mismatch $\Delta k$ by designing a negative electrostatic spring and tuning the stiffness as described in section IV.

3) Design the resonator with large ratio between the in-phase and anti-phase frequencies.

\section{CONCLUSION}

In this paper we investigated the dissipation of energy through the substrate in a non-ideal anti-phase operated tuning fork MEMS resonators. A closed form mathematical model was developed for Q-factor of non-ideal resonators as a function of stiffness imbalance. Reduction of the structural imbalances for Q-factor maximization was proposed based on post-fabrication electrostatic tuning of stiffness. The model was validated using a vacuum packaged SOI MEMS tuning fork with an initial operational frequency of $2.2 \mathrm{kHz}$ and Q-factor of 0.6 million. By electrostatically tuning one of the suspension springs, momentum imbalance caused by the fabrication imperfections was minimized, suppressing anchor loss and increasing the Q-factor to above 0.8 million. The substrate dissipation model, validated by experiments, is a valuable design tool, as well as post-fabrication for predicting the trimming and tuning of Q-factors in anti-phase resonant MEMS.

\section{REFERENCES}

[1] A. A. Trusov, I. P. Prikhodko, S. A. Zotov, and A. M. Shkel, "Lowdissipation silicon MEMS tuning fork gyroscopes for rate and whole angle measurements," IEEE Sensors J., vol. 11, no. 11, pp. 2763-2770, Nov. 2011.

[2] R. Hopkins, J. Miola, W. Sawyer, R. Setterlund, and B. Dow, "The silicon oscillating accelerometer: A high-performance MEMS accelerometer for precision navigation and strategic guidance applications," in Proc. Nat. Tech. Meeting Inst. Navigat., San Diego, CA, USA, Jan. 2005, pp. 970-979.

[3] D. D. Lynch, "Coriolis vibratory gyros," in Proc. Symp. Gyro Technol., Stuttgart, Germany, Sep. 1998, pp. 1.0-1.14.

[4] S. A. Zotov, A. A. Trusov, and A. M. Shkel, "High-range angular rate sensor based on mechanical frequency modulation," J. Microelectromech. Syst., vol. 21, no. 2, pp. 398-405, Apr. 2012.

[5] R. L. Borwick et al., "A high Q, large tuning range MEMS capacitor for RF filter systems," Sens. Actuators A, Phys., vol. 103, nos. 1-2, pp. 33-41, Jan. 2003.

[6] M. S. Weinberg, B. T. Cunningham, and C. W. Clapp, "Modeling flexural plate wave devices," J. Microelectromech. Syst., vol. 9, no. 3, pp. 370-379, Sep. 2000.

[7] N. Yazdi, F. Ayazi, and K. Najafi, "Micromachined inertial sensors," Proc. IEEE, vol. 86, no. 8, pp. 1640-1659, Aug. 1998.

[8] M. Weinberg, R. Candler, S. Chandorkar, J. Varsanik, T. Kenny, and A. Duwel, "Energy loss in MEMS resonators and the impact on inertial and RF devices," in Proc. Solid-State Sensors, Actuat. Microsyst. Conf., Transducers, Denver, CO, USA, Jun. 2009, pp. 688-695.

[9] C. Zener, "Internal friction in solids," Proc. Phys. Soc., vol. 52, no. 1, pp. $152-166,1940$

[10] C. Zener, "Internal friction in solids II. General theory of thermoelastic internal friction," Phys. Rev., vol. 53, no. 1, pp. 90-99, 1938.

[11] M. F. Zaman, A. Sharma, and F. Ayazi, "High performance matchedmode tuning fork gyroscope," in Proc. MEMS, Istanbul, Turkey, Jan. 2006, pp. 66-69.

[12] A. Duwel, M. Weinstein, J. Gorman, J. Borenstein, and P. Ward, "Quality factors of MEMS gyros and the role of thermoelastic damping," in Proc. 15th IEEE Int. Conf. Micro Electro Mech. Syst., Jan. 2002, pp. 214-219.

[13] M. Lutz et al., "A precision yaw rate sensor in silicon micromachining," in Proc. Transducers, Chicago, IL, USA, Jun. 1997, pp. 847-850.

[14] J. Geen, "Coupling for multiple masses in a micromachined device," U.S. Patent 5635638, Jun. 3, 1997.
[15] A. A. Trusov, A. R. Schofield, and A. M. Shkel, "Micromachined rate gyroscope architecture with ultra-high quality factor and improved mode ordering," Sens. Actuators A, Phys., vol. 165, pp. 26-34, Jan. 2011.

[16] A. A. Trusov, A. R. Schofield, and A. M. Shkel, "A substrate energy dissipation mechanism in in-phase and anti-phase micromachined $z$-axis vibratory gyroscopes," J. Micromech. Microeng., vol. 18, pp. 1-10, Sep. 2008.

[17] A. A. Trusov, S. A. Zotov, and A. M. Shkel, "Electrostatic regulation of quality factor in tuning fork MEMS," in Proc. IEEE Sensors Conf., Limerick, Ireland, Oct. 2011, pp. 20-23.

[18] P. Cheng, Y. Zhang, W. Gu, and Z. Hao, "Effect of polarization voltage on the measured quality factor of a multiple-beam tuning-fork gyroscope," Sens. Actuators A, Phys., vol. 187, pp. 118-126, Nov. 2012.

[19] M. D. Greenberg, Advanced Engineering Mathematics, 2nd ed. Englewood Cliffs, NJ, USA: Prentice-Hall, 1998.

[20] V. I. Arnold, Ordinary Differential Equations. Cambridge, MA, USA: MIT Press, 1978.

[21] M. A. Hopcroft, W. D. Nix, and T. W. Kenny, "What is the Young's modulus of silicon?" J. Microelectromech. Syst., vol. 19, no. 2, pp. 229-238, Apr. 2010.

[22] A. Duwel, R. N. Candler, T. W. Kenny, and M. Varghese, "Engineering MEMS resonators with low thermoelastic damping," J. Microelectromech. Syst., vol. 15, no. 6, pp. 1437-1445, Dec. 2006.

[23] S. Prabhakar and S. Vengallatore, "Theory of thermoelastic damping in micromechanical resonators with two-dimensional heat conduction," J. Microelectromech. Syst., vol. 17, no. 2, pp. 494-502, Apr. 2008.

[24] D. S. Bindel and S. Govindjee, "Elastic PMLs for resonator anchor loss simulation," Int. J. Numer. Methods Eng., vol. 64, no. 6, pp. 789-818, Oct. 2005.

[25] B. R. Simon, A. A. Trusov, and A. M. Shkel, "Anti-phase mode isolation in tuning-fork MEMS using a lever coupling design," in Proc. IEEE Sensors Conf., Taipei, Taiwan, Oct. 2012, pp. 42-45.

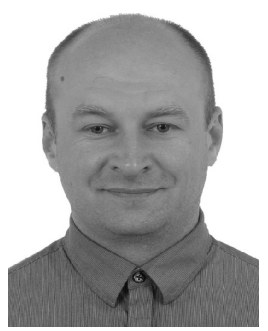

Sergei A. Zotov received the M.S. and Ph.D. degrees in mechanical engineering and control systems from Tula State University, Tula, Russia, in 1999 and 2002, respectively. He is currently a Lead Engineer in the MicroSystems and MicroFluidics Laboratory at General Electric's Global Research Center. From 2008 to 2014, he was a Post-Doctoral Scientist with the MicroSystems Laboratory at University of California, Irvine, CA, USA, where he was responsible for the development, design, fabrication, and testing of micromachined devices and systems for inertial navigation. Over the last 12 years, his focus has been on the research and development of MEMS gyroscopes and accelerometers. $\mathrm{He}$ has authored eight peer-reviewed journal articles and more than 20 international conference papers in the field of inertial MEMS. He holds eight Russian patents, two U.S. patents, and three U.S. patents pending on inertial MEMS. He was a recipient of the Gold Medal at the 2004 International Salon of Inventions in Geneva, Switzerland, the Outstanding Paper Award at the 2011 Transducers Conference, and the Best Paper Award at the 2012 IMAPS Device Packaging Conference. He is a Reviewer for major MEMS journals.

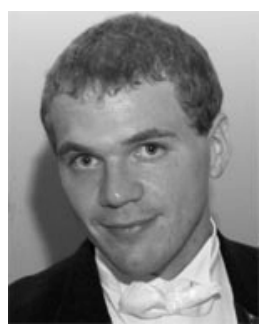

Brenton R. Simon received the B.S. degree in mechanical engineering from the University of Cincinnati, Cincinnati, OH, USA, and the M.S. degree in mechanical and aerospace engineering from the University of California, Davis, in 2007 and 2009, respectively. $\mathrm{He}$ is currently pursuing the Ph.D. degree at the University of California, Irvine, as a member of the UC Irvine MicroSystems Laboratory, where he is involved in the advancement of microfabricated inertial sensors through design, fabrication, and characterization. He has authored over 10 peer-reviewed journal and conference publications in the field of inertial MEMS sensors. He holds two pending U.S. patents. 


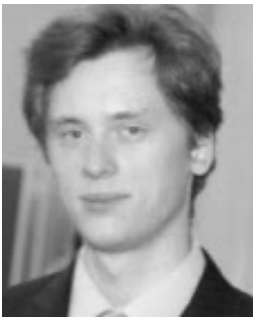

Igor P. Prikhodko (S'09-M'13) is a MEMS Advanced Development Engineer with Analog Devices, Inc., where he is involved in research and development of advanced inertial MEMS sensors. He received the B.S. (cum laude) degree in mechanics and mathematics from Moscow State University, Moscow, Russia, and the M.S. and Ph.D. degrees in mechanical and aerospace engineering from the University of California, Irvine, CA, USA, in 2007, 2008 , and 2013, respectively. He has authored over 30 peer-reviewed journal and refereed conference papers. He holds one issued U.S. patent and one U.S. patent pending on inertial MEMS. He was a recipient of the Outstanding Paper Award at the 2011 Transducers Conference and the Best Paper Award at the 2012 IMAPS Device Packaging Conference. He serves as a Reviewer for major MEMS journals and on program committees for the IEEE International Symposium on Inertial Sensors and Systems and the International Symposium on Microelectronics. $\mathrm{He}$ is a member of the American Society of Mechanical Engineers.



Alexander A. Trusov (M’06) is a Senior Research Scientist with Northrop Grumman Corporation, where he is involved in research and development of advanced navigation sensors, instruments, and systems. He received the B.S. and M.S. degrees in applied mathematics and mechanics from Moscow State University, Moscow, Russia, in 2004, and the M.S. and Ph.D. degrees in mechanical and aerospace engineering from the University of California, Irvine (UCI), CA, USA, in 2006 and 2009, respectively. From 2009 to 2013, he was a Project Scientist with the Mechanical and Aerospace Department, UCI, where he served as the PI and a Co-PI on more than six DoD sponsored projects. His current research interests include design, modeling, fabrication, and vacuum packaging of micromachined inertial systems, sensor and instrument self-calibration, design of characterization experiments, and statistical data processing and analysis. He has authored over 70 journal and conference papers and holds seven issued U.S. patents. He was a recipient of the Outstanding Paper Award at 2011 Transducers Conference, the Design Contest Award at the 2011 System-on-Chip Conference, and the Best Paper Award at the 2012 IMAPS Device Packaging Conference.
He currently serves on program committees for the Saint Petersburg International Conference on Integrated Navigation Systems, the IEEE International Symposium on Inertial Sensors and Systems, and the IEEE/ION Position Location and Navigation Symposium. He is a member of the American Society of Mechanical Engineers and the Institute of Navigation.

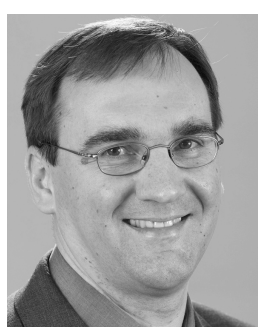

Andrei M. Shkel (S'95-A'98-SM'08-F'14) received the Diploma (Hons.) degree in mechanics and mathematics from Moscow State University, Russia, and the Ph.D. degree in mechanical engineering from the University of Wisconsin, Madison, WI, USA, in 1991 and 1997, respectively. In 2000, he joined the Faculty of the University of California, Irvine, where he is currently a Professor with the Department of Mechanical and Aerospace Engineering, with a joint appointment with the Department of Electrical Engineering and Computer Science and the Department of Biomedical Engineering.

From 2009 to 2013, he served as a Program Manager with the Microsystems Technology Office, Defense Advanced Research Projects Agency (DARPA), Arlington, VA, USA, where he created and managed a comprehensive portfolio of programs focused on microtechnology for positioning, navigation, and timing applications, including the nationwide programs Micro Rate Integrating Gyroscope, Primary and Secondary Calibration on Active Layer, Timing and Inertial Measurement Unit, and Chip-Scale Combinatorial Atomic Navigator. His professional interests, reflected in over 180 publications and two books, include solid-state sensors and actuators, MEMS-based neuroprosthetics, sensor-based intelligence, and control theory. He holds 26 U.S. and worldwide patents (12 are pending) on micromachined angle-measuring gyroscopes, widebandwidth rate gyroscopes, design and fabrication of light manipulators and tunable optical filters, and hybrid micromachining processes. His current research interests include the design, manufacturing, and advanced control of microelectromechanical systems for biomedical and inertial navigation applications, in particular, on the development of high-precision micromachined gyroscopes. He served on a number of editorial boards, most recently as an Editor of the JOURNAL OF Microelectromechanical Systems, an Editorial Board Member for the International Journal on Smart Structures and Systems, Founding Chair of the IEEE International Symposium on Inertial Sensors and Systems, a TPC Member of Hilton Head 2009, and General Chair of the 2005 IEEE Sensors Conference. He was a recipient of the IEEE Sensors Council Technical Achievement Award in 2009, the NSF CAREER Award in 2005, the George E. Brown, Jr. Award in 2002, and the Best Faculty Research Award in 2006

Dr. Shkel was a recipient of the Office of the Secretary of Defense Medal for Exceptional Public Service for his work at DARPA as a Program Manager in 2013. He is the IEEE Fellow. 